\title{
Kalmar - A Heartsafe Community
}

\section{Purpose}

The purpose of this project was to spread information about the importance of alerting, starting CPR and knowing where public automated external defibrillators (AEDs) are located, as well as creating a public interest in CPR education in our hometown Kalmar.

\section{Material and methods}

We reviewed all local AEDs registered in the national AED registry and examined routines for CPR education. Only a few AEDs were available outside office hours, and CPR education was unorganized.

This led us to starting a non-profit organization, called "Kalmar - A Heartsafe Community" that we run during our spare time.

We have initiated a cooperation with local companies, local profiles, the municipality and the county council for sponsorship.

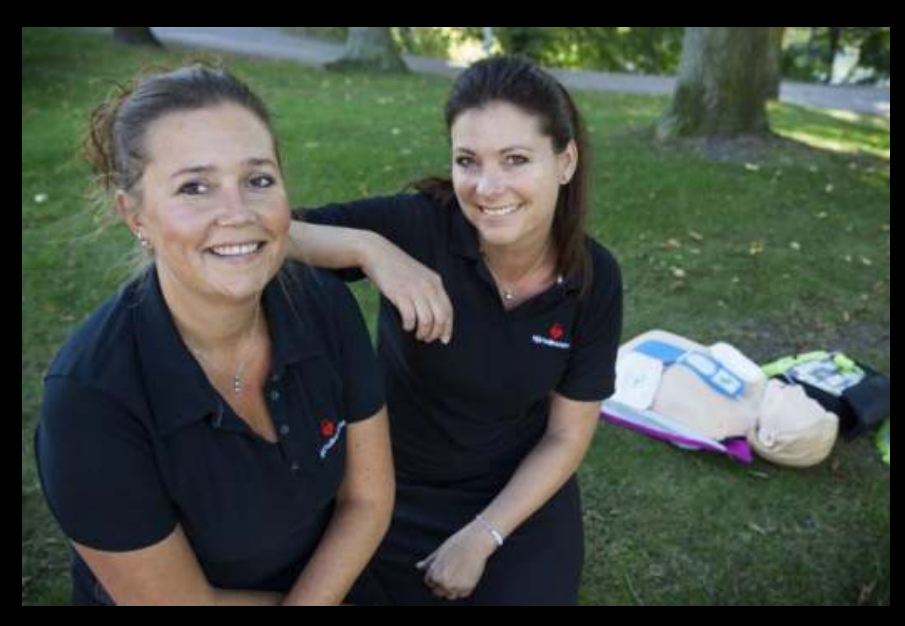

Therése Johansson, Department of Cardiology, Kalmar County Council, Kalmar, Sweden, Nina Berg, Department of Emergency Care, Kalmar County Council, Kalmar, Sweden

\section{Results}

An information film highlighting the importance of early alarms, CPR and the use of AEDs, is broadcasted in public transportations, malls and in waiting rooms of the county council.

Commercials are published in newspapers, radio, and social media. So far, more than 400 people have received basic CPR education.

The sponsorships have been used to set up three public AEDs, equipped with an alarm system that alerts the emergency services.

\section{Conclusions}

The project has contributed to more people educated in CPR and more available AEDs in Kalmar. Moreover, it has helped creating a public interest regarding how to treat a cardiac arrest (CA). We believe that this subsequently will lead to an increased chance of surviving a CA in Kalmar. 